\title{
Genetic variation in Aquaporin-4 moderates the relationship between sleep and brain $A \beta$-amyloid burden
}

Stephanie R. Rainey-Smith ${ }^{1,2}$, Gavin N. Mazzucchelli ${ }^{3}{ }^{3}$, Victor L. Villemagne ${ }^{4,5}$, Belinda M. Brown 1,2,6, Tenielle Porter ${ }^{3,7}$,

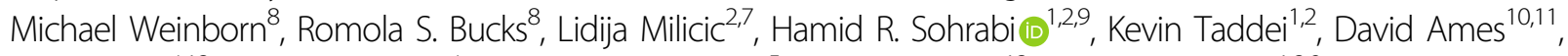
Paul Maruff ${ }^{4,12}$, Colin L. Masters ${ }^{4}$, Christopher C. Rowe', Olivier Salvado ${ }^{13}$, Ralph N. Martins ${ }^{1,2,9}$ and

Simon M. Laws $\mathbb{1}^{3,7,14}$, for the AIBL Research Group

\begin{abstract}
The glymphatic system is postulated to be a mechanism of brain A $\beta$-amyloid clearance and to be most effective during sleep. Ablation of the astrocytic end-feet expressed water-channel protein, Aquaporin-4, in mice, results in impairment of this clearance mechanism and increased brain AB-amyloid deposition, suggesting that Aquaporin-4 plays a pivotal role in glymphatic function. Currently there is a paucity of literature regarding the impact of AQP4 genetic variation on sleep, brain $A \beta$-amyloid burden and their relationship to each other in humans. To address this a cross-sectional observational study was undertaken in cognitively normal older adults from the Australian Imaging, Biomarkers and Lifestyle (AIBL) study. Genetic variants in AQP4 were investigated with respect to self-reported Pittsburgh Sleep Quality Index sleep parameters, positron emission tomography derived brain A $\beta$-amyloid burden and whether these genetic variants moderated the sleep-A $\beta$-amyloid burden relationship. One AQP4 variant, rs72878776, was associated with poorer overall sleep quality, while several SNPs moderated the effect of sleep latency (rs491148, rs9951307, rs7135406, rs3875089, rs151246) and duration (rs72878776, rs491148 and rs2339214) on brain A -amyloid burden. This study suggests that AQP4 genetic variation moderates the relationship between sleep and brain A $\beta$ amyloid burden, which adds weight to the proposed glymphatic system being a potential $A \beta$-amyloid clearance mechanism and suggests that AQP4 genetic variation may impair this function. Further, AQP4 genetic variation should be considered when interpreting sleep-A $\beta$ relationships.
\end{abstract}

\section{Introduction}

Estimates suggest that dysfunctional sleep may be present in up to $45 \%$ of Alzheimer's disease (AD) patients ${ }^{1}$; manifesting commonly as frequent awakenings, increased sleep latency (time to fall asleep) and poor sleep

Correspondence: Simon M Laws (s.laws@ecu.edu.au)

${ }^{1}$ Centre of Excellence for Alzheimer's Disease Research and Care, School of Medical and Health Sciences, Edith Cowan University, Joondalup 6027 WA, Australia

${ }^{2}$ Sir James McCusker Alzheimer's Disease Research Unit, Hollywood Private Hospital, Perth 6009 WA, Australia

Full list of author information is available at the end of the article

These authors jointly supervised this work: Stephanie R. Rainey-Smith and Gavin N. Mazzucchelli

AIBL Research Group: http://aibl.csiro.au/about/aibl-research-team maintenance ${ }^{2}$. However, accumulating evidence also suggests that there is a bi-directional relationship between sleep and $\mathrm{AD}$ phenotypes ${ }^{3-5}$; i.e., in addition to the $\mathrm{AD}$ phenotype leading to sleep dysfunction, dysfunctional sleep contributes to the AD phenotype.

$\mathrm{A} \beta$-amyloid $(\mathrm{A} \beta)$ accumulates gradually in the brain of individuals as they progress towards a diagnosis of $\mathrm{AD}^{6}$. This accumulation of $A \beta$ is thought to begin about 20 years before the onset of $\mathrm{AD}$ symptomology ${ }^{7}$, and in the sporadic form of the disease, is hypothesized to be driven by poor clearance mechanisms ${ }^{6}$. The mechanisms of $A \beta$ clearance from the human brain are multiple, with some 
specific factors involved yet to be fully understood ${ }^{8}$. Significantly, however, it has been observed in mice that good quality sleep enhances brain $A \beta$ clearance ${ }^{9}$, while dysfunctional sleep exacerbates $A \beta$ accumulation in humans ${ }^{10}$ and animal models ${ }^{11}$. Further, we have recently shown that in cognitively normal older adults, increased sleep latency is associated with higher brain $A \beta$ burden ${ }^{12}$. It is hypothesized that the brain has a lymphatic-like clearance system that operates parallel to the human lymphatic system through the employment of a network of paravascular clearing mechanisms ${ }^{13}$. This lymphaticlike clearance system has been termed the glymphatic system $^{14,15}$, and is postulated to function almost entirely during sleep ${ }^{9}$.

Evidence from animal models supports a cerebral perivascular and paravascular clearing mechanism that involves the bulk flow of interstitial fluid and the involvement of the water-channel protein, Aquaporin-4 $(\mathrm{AQP} 4)^{16}$, located primarily in the subpial and perivascular end-feet of astrocytic processes. Further, evidence from Aqp4 gene knockout mice supports the notion that the functionality of AQP4 is related to the efficacy of $A \beta$ clearance $^{17}$, likely via the glymphatic system ${ }^{18}$. Additionally, a study of autopsied human brains observed that AQP4 immunoreactivity is distributed in a manner similar to neuritic $\mathrm{A} \beta$ plaques ${ }^{19}$; suggesting that $\mathrm{AQP} 4$ is likely to be linked to $A \beta$ plaque deposition in the brain ${ }^{20}$. Further, a decrease in AQP4 expression or loss of perivascular localization could contribute to reduced $A \beta$ clearance ${ }^{21}$.

Rare in silico-predicted functional variants have been identified in human $A Q P 4$, which have been shown to impair water permeability in vitro ${ }^{22}$. However, no studies to date have investigated the role of $A Q P 4$ genetic variation in $\mathrm{AD}$, sleep and $\mathrm{A} \beta$ clearance. To deepen our understanding of the role of AQP4 in $\mathrm{AD}$, we investigated genetic variation across the $A Q P 4$ gene with respect to the relationship with, and between, sleep quality/quantity and brain $A \beta$ burden. We hypothesized that poorer quality sleep would be associated with higher $A \beta$ brain burden and that genetic variants in $A Q P 4$ would moderate this relationship. This hypothesis was derived from the premise that both poor sleep quality decreases $A \beta$ clearance (i.e., results in higher brain $A \beta$ burden), and also that suboptimal glymphatic clearance during dysfunctional sleep will result in a higher cerebral A $\beta$ burden; ergo, AQP4 genetic variation would have a functional impact on brain A $\beta$ burden.

\section{Materials and methods}

\section{Study participants}

This cross-sectional investigation utilized data collected from Cognitively Normal $(\mathrm{CN})$ older adults of the Australian Imaging, Biomarkers and Lifestyle (AIBL) Study; a prospective longitudinal study of ageing launched in 2006.
All volunteers were aged 60 years and above at baseline. Further details regarding the design, enrolment process, neuropsychological assessments, and diagnostic criteria of the AIBL Study have been previously described elsewhere $^{23}$. The AIBL Study is approved by the institutional ethics committees of Austin Health, St Vincent's Health, Hollywood Private Hospital, and Edith Cowan University (ECU), and informed written consent was given by all volunteers.

\section{Sleep measures}

Subjective sleep quality and disturbances were assessed via the Pittsburgh Sleep Quality Index (PSQI) ${ }^{24}$ in $462 \mathrm{CN}$ older adults at the 72-month time point of the study. Several parameters are subsequently derived from this 19item self-report measure, including sleep quality, latency (in $\mathrm{min}$ ), duration (reported in hours), efficiency, sleep disturbance, medication use and daytime dysfunction. A further global score of sleep quality, PSQI Total, is also derived, with a score $>5$ indicating poor sleep. For the present study, analyses focused on parameters related to night-time function and thus, the factors studied herein were limited initially to overall sleep quality (PSQI Total), then subsequently to the sub-scales of sleep latency, sleep duration, sleep efficiency, and sleep disturbances.

\section{Brain imaging}

Of these 462 participants, 222 also underwent $A \beta$ imaging. $A \beta$ imaging was performed via positron emission tomography (PET) using one of the following radiolabeled $\mathrm{A} \beta$ tracers; ${ }^{11} \mathrm{C}$-Pittsburgh Compound $\mathrm{B}(\mathrm{PiB}),{ }^{18} \mathrm{~F}-$ florbetapir or ${ }^{18} \mathrm{~F}$-flutemetamol, as previously described $^{25-27}$. Images were analyzed using CapAIBL, a webbased freely available magnetic resonance (MR)-less methodology, to generate PET standardized uptake value (SUV) ratios (SUVR) for all tracers ${ }^{28}$. Briefly, SUVs were summed and normalized to either the cerebellar cortex SUV (PiB), whole cerebellum SUV (florbetapir), or pons SUV (flutemetamol), to yield the target-region to reference-region SUVR. To allow for the analysis of these different tracers as a single continuous variable, a linear regression transformation was applied to generate $\mathrm{PiB}$ like SUVR units termed the 'Before the Centiloid Kernel Transformation' (BeCKeT) scale ${ }^{29}$. PiB SUVR and florbetapir/flutemetamol BeCKeT were utilized in this crosssectional study.

\section{Genetic data}

Genetic data were derived from a genome-wide singlenucleotide polymorphism (SNP) array conducted on the Illumina OmniExpressHumanExome + BeadChip with subsequent imputation using impute2 ver2.3, with the 1000 genome reference panel (2015 release). SNP data 
from the AQP4 genomic region (GRCh37 Chr18:24,432,000-24,446,000) were extracted and subjected to quality control in GoldenHelix SNP and Variation Suite (SVS version 8.7.1), which included removal of SNPs with call rate $<95 \%$, Minor allele frequency $<5 \%$ and departure from Hardy-Weinberg Equilibrium $(p<$ 0.05), leaving 32 SNPs (Supplementary Table 1). After Linkage Disequilibrium (LD) pruning $\left(r^{2}\right.$ cutoff of 0.8 , window size 10, increment 5), $13 A Q P 4$ SNPs were selected to provide full coverage of the gene (Supplementary Table 1, Supplementary Figure 1). Apolipoprotein $\mathrm{E}(A P O E)$ genotype, specifically the presence of the $\varepsilon 4$ allele, the major genetic risk factor for $\mathrm{AD}$, was determined using TaqMan genotyping assays (Life Technologies, USA) for rs7412 (Assay ID: C__904973_10) and rs429358 (Assay ID: C__3084793_20) on a QuantStudio $12 \mathrm{~K}$ Flex real-time PCR system (Applied Biosystems, USA).

\section{Statistical analysis}

Statistical analyses were carried out in either Golden Helix (Inc.) SVS (version 8.7.1) for linear regression analyses, using additive (homozygote for the minor allele $(\mathrm{MM})$ vs. heterozygote for the minor allele $(\mathrm{Mm})$ vs. homozygote for the major allele $(\mathrm{mm})$ ), recessive (homozygote for the minor allele (MM) vs. heterozygote/ homozygote for the major allele $(\mathrm{Mm} / \mathrm{mm}))$ and dominant (heterozygote/homozygote for the minor allele (Mm or $\mathrm{MM})$ vs. homozygote for the major allele $(\mathrm{mm})$ ) genetic models, or IBM SPSS Statistics, Version 24.0 (IBM Corp., Armonk, NY) for moderation analyses, using recessive and dominant models only. Nominal significance (uncorrected) was reported at $p<0.05$. However, final levels of significance were ascertained after correction for the False Discovery Rate (FDR) with significance threshold set at $q<0.05^{30}$. Linear regression analysis, with respect to $A \beta$ burden, included the covariates of age, sex, and $A P O E$ genotype (presence/absence of the $\varepsilon 4$ allele). For the PSQI sleep parameters, body mass index (BMI), depressive symptomology (Geriatric depression Scale; GDS) and a medical history of cardiovascular disease (CVD) were also included as covariates. The relationship between $A Q P 4$ SNPs and PSQI sleep parameters was undertaken using a two-stage approach. First, the association with overall sleep quality (PSQI total) was assessed followed by analysis of association with the PSQI subscales (sleep latency, duration, efficiency and disturbances).

Moderation analysis in SPSS utilized a custom dialog: PROCESS (release 2.16.3) ${ }^{31}$ with 5000 bias-corrected and accelerated bootstrap samples, with $95 \%$ confidence intervals. AQP4 SNPs were included as the moderator variable $(W)$, brain $\mathrm{A} \beta$ burden as the outcome variable $(Y)$, with each of the five selected PSQI sleep parameters entered individually as the independent variable $(X)$. Moderation analyses covaried for age, BMI, medical history of CVD, GDS and APOE \&4 allele carriage as previously reported ${ }^{12}$. Post hoc simple slopes analysis was used to visualize the moderation of the effect of $X$ on $Y$ by the moderating variable, $W^{32}$

\section{Results}

Demographic characteristics for the study cohort are presented in Table 1. Neuroimaging data were only available in $222 \mathrm{CN}$ older adults at the same assessment time point at which the PSQI was administered. However, there were no significant differences in terms of the distributions or means of the demographic variables between the PSQI only group $(n=462)$ and the PSQI plus imaging subset $(n=222)$.

\section{AQP4 genetic variation and PSQI sleep parameters or brain $A \beta$ burden}

Linear regression analysis was performed to determine whether $A Q P 4$ SNPs were associated firstly with overall sleep quality (PSQI total) and subsequently with the PSQI sub-scales (sleep latency, sleep duration, sleep efficiency, and sleep disturbances), using both a base statistical model (no covariates) and an adjusted statistical model, covarying for age, BMI, medical history of CVD, GDS and

Table 1 Cohort demographics

\begin{tabular}{|c|c|c|}
\hline & PSQI Only & PSQI and $A \beta$ \\
\hline$n$ & 462 & 222 \\
\hline Age, years & $75.0 \pm 6.0$ & $75.2 \pm 6.1$ \\
\hline Sex, \% Female & 58.1 & 57.2 \\
\hline$A P O E, \%$ \&4 carriers & 22.7 & 23 \\
\hline$A \beta\left(S U V R / B e C K e T^{\mathrm{a}}\right)$ & $1.38 \pm 0.38^{\mathrm{b}}$ & $1.38 \pm 0.38$ \\
\hline Time between PSQI and PET scan (days) & & $173.7 \pm 132.3$ \\
\hline MMSE & $28.9 \pm 1.3$ & $28.9 \pm 1.4$ \\
\hline BMI $\left(\mathrm{kg} / \mathrm{m}^{2}\right)$ & $26.5 \pm 4.3$ & $26.4 \pm 4.2$ \\
\hline GDS & $1.4 \pm 1.7$ & $1.3 \pm 1.6$ \\
\hline$\%$ Good sleepers ${ }^{c}(n)$ & $50.9(235)$ & $55.9(124)$ \\
\hline PSQI Total & $6.2 \pm 1.2$ & $5.6 \pm 3.2$ \\
\hline Sleep latency (minutes) & $19.9 \pm 19.4$ & $17.0 \pm 16.6$ \\
\hline Sleep duration (hours) & $6.8 \pm 1.2$ & $7.0 \pm 1.2$ \\
\hline
\end{tabular}

${ }^{a 11} \mathrm{C}$-Pittsburgh compound B PET (PiB-PET) like standardized uptake value ratio (SUVR) generated using the Before the Centiloid Kernel Transformation (BeCKeT) scale

b $n=222$

'Good sleeper, defined by PSQI Total score $\leq 5$

All values represented as mean \pm s.d., unless otherwise indicated. $A \beta A \beta$ amyloid; $A P O E$ apolipoprotein $\mathrm{E} \varepsilon 4$ allele carriage; $B M I$ body mass index; GDS Geriatric Depression Scale; MMSE Mini Mental State Examination; PET Positron Emission Tomography; PSQI Pittsburgh Sleep Quality Index 
Table 2 Association of AQP4 SNPs with Pittsburgh Sleep Quality Index sleep parameters

\begin{tabular}{|c|c|c|c|c|c|c|c|}
\hline \multirow{2}{*}{$\begin{array}{l}\text { PSQI sleep } \\
\text { parameter }\end{array}$} & \multirow[t]{2}{*}{ SNP Ref } & \multicolumn{2}{|c|}{ Additive $^{\mathrm{a}}$} & \multicolumn{2}{|c|}{ Dominant $^{a}$} & \multicolumn{2}{|c|}{ Recessive $^{a}$} \\
\hline & & Base $^{\mathrm{b}}$ & $\operatorname{Adj}^{\mathbf{b}}$ & Base $^{b}$ & $A d j^{\mathbf{b}}$ & Base $^{\mathrm{b}}$ & $\operatorname{Adj}^{\mathbf{b}}$ \\
\hline \multirow[t]{3}{*}{ PSQI Total } & rs71353406 & 0.130 & 0.100 & 0.042 & 0.045 & 0.856 & 0.871 \\
\hline & rs72878776 & 0.593 & 0.836 & 0.647 & 0.466 & $0.001^{c}$ & $0.002^{c}$ \\
\hline & rs3875089 & 0.494 & 0.442 & 0.940 & 0.931 & 0.012 & 0.021 \\
\hline $\begin{array}{l}\text { Sleep } \\
\text { disturbances }\end{array}$ & rs68006382 & 0.097 & 0.146 & 0.034 & 0.077 & 0.062 & 0.902 \\
\hline
\end{tabular}

${ }^{a} G$ enetic models: Additive (homozygote for the minor allele (MM) vs. heterozygote for the minor allele $(\mathrm{Mm})$ vs. homozygote for the major allele $(\mathrm{mm})$ ); Recessive (homozygote for the minor allele (MM) vs. heterozygote/ homozygote for the major allele $(\mathrm{Mm} / \mathrm{mm})$ ); Dominant (heterozygote/homozygote for the minor allele ( $\mathrm{Mm}$ or $\mathrm{MM})$ vs. homozygote for the major allele $(\mathrm{mm}))$

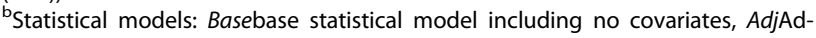
justed statistical model (covaries for: age, sex, body mass index (BMI), geriatric depression scale (GDS) and a medical history of CVD). Values that reached nominal significance $(p<0.05$, uncorrected) are bolded

$c^{c}$ values significant after False Discovery Rate correction $(q<0.05)$

Summary of Aquaporin-4 (AQP4) SNPs demonstrating significant associations with sleep parameters. PSQI Pittsburgh Sleep Quality Index Sleep Parameters: PSQI Total, sleep disturbances. SNP Ref, reference single-nucleotide polymorphism marker (rs); AQP4 Aquaporin-4

$A P O E$ \&4 allele carriage. Nominal significance (Table 2) was observed with respect to PSQI Total (rs71353406, rs72878776, and rs3875089) and subsequently, in the subscale analyses, with sleep disturbances (rs68006382). Of these associations, only that of rs72878776 with PSQI Total (base model, $\beta=4.74$ (s.e.: 1.37), $p=0.0006$; adjusted model, $\beta=4.15$ (s.e.: 1.34), $p=0.002$ ) remained significant after FDR correction (base model, $q=0.008$; adjusted model, $q=0.028$ ). No further associations were observed for remaining genetic variants and PSQI sleep parameters (Supplementary Table 2).

Linear regression analyses were also performed to determine whether AQP4 SNPs were associated with brain $\mathrm{A} \beta$ burden, again using both a base statistical model (no covariates) and an adjusted statistical model, covarying for age, sex and $A P O E \& 4$ allele carriage. No significant associations were observed between $A Q P 4$ genetic variants and brain $\mathrm{A} \beta$ burden either independently or when the covariates of age, sex, and APOE \&4 were included in the adjusted models (Supplementary Table 3).

\section{AQP4 moderation of PSQI sleep parameter-brain $A \beta$ burden relationship}

Linear regression analysis (Supplementary Table 4), excluding AQP4 SNPs, revealed that sleep latency (minutes) was associated with $\mathrm{A} \beta$ burden $(\beta=0.004, t(215)=$ 2.66; 95\% CI, 0.001-0.007; $p=0.008)$, consistent with a previous report in a subset of this same sample ${ }^{12}$. No other PSQI sleep parameter was observed to be associated with brain $\mathrm{A} \beta$ burden in our analyses.
To determine whether AQP4 SNPs moderated the relationship between the 5 PSQI sleep parameters and brain $A \beta$ burden, multivariate linear regression analyses were performed within the moderation model. Nine of these statistical models revealed significant moderation effects for AQP4 SNPs on the PSQI sleep parameters of sleep latency and sleep duration (Table 3). The relationship between PSQI-determined sleep latency and brain $\mathrm{A} \beta$ burden was observed to be significantly moderated by a total of five $A Q P 4$ SNPs. The interaction of the AQP4 SNPs rs491148 and sleep latency was statistically significant for both dominant $\left(R^{2}\right.$-change $\left(\Delta R^{2}\right)=0.017 ; p=$ $0.036)$ and recessive $\left(\Delta R^{2}=0.020 ; p=0.022\right)$ genetic models. While rs9951307 $\left(\Delta R^{2}=0.015 ; p=0.048\right)$, rs71353406 $\left(\Delta R^{2}=0.019 ; p=0.030\right), \operatorname{rs} 3875089\left(\Delta R^{2}=\right.$ $0.019 ; p=0.028)$ and $\operatorname{rs} 151246\left(\Delta R^{2}, 0.039 ; p=0.002\right)$ were significant in the dominant genetic model only. Post hoc simple slopes analyses (Fig. 1) revealed that for rs3875089, rs71353406, and rs491148, carriage of at least one copy of the minor allele was associated with higher brain $\mathrm{A} \beta$ burden as sleep latency increased, while for rs9951307 and rs151246 this relationship was observed for homozygosity of the major allele. All results from moderation analyses are presented in Supplementary Tables 5-9.

Three AQP4 SNPs interacted with PSQI-determined sleep duration (in hours) to significantly impact brain $A \beta$ burden, namely rs72878776, rs2339214 and rs491148 (Table 3). For rs72878776 $\left(\Delta R^{2}=0.019 ; p=0.034\right)$ and rs491148 $\left(\Delta R^{2}=0.016 ; p=0.045\right)$ the association was observed in the dominant genetic model, while for rs2339214 $\left(\Delta R^{2}=0.041 ; p=0.002\right)$, the association was observed in the recessive model. Post hoc simple slopes analyses (Fig. 2) revealed that for rs72878776 and rs491148, carriage of at least one copy of the minor allele resulted in higher brain $A \beta$ burden with a shorter duration of sleep. However, the opposite was observed for rs2339214, where homozygosity of the minor allele resulted in higher brain $\mathrm{A} \beta$ burden with a longer duration of sleep.

\section{Discussion}

The primary aim of this study was to determine whether genetic variation within $A Q P 4$ moderated the relationship between PSQI-derived self-reported sleep quality and brain $\mathrm{A} \beta$ burden as assessed by PET in cognitively normal older adults of the AIBL Study. This study is the first to report genetic variation in $A Q P 4$ to be both associated with altered, self-reported, 'overall' sleep quality (PSQI Total score), and to moderate the relationship between the sleep parameters of latency (time taken to fall asleep), duration (length of sleep), and brain $A \beta$ burden.

The association of sleep latency with increased brain $A \beta$ burden confirms results previously reported in a subset $(n$ 
Table 3 Moderation analysis for AQP4 SNPs on sleep latency and sleep duration

\begin{tabular}{|c|c|c|c|c|c|c|c|c|c|c|c|c|}
\hline & \multicolumn{6}{|c|}{ Dominant $^{\mathrm{a}}$} & \multicolumn{6}{|c|}{ Recessive $^{a}$} \\
\hline & $\beta$ & s.e. & Sig. & $R^{2}$ & Sig. & $\Delta R^{2}$ & $\beta$ & s.e. & Sig. & $R^{2}$ & Sig. & $\Delta R^{2}$ \\
\hline \multicolumn{13}{|l|}{ LATENCY } \\
\hline Model summary: & : rs151246 & & & 0.201 & $<0.001$ & & & & & 0.165 & $<0.001$ & \\
\hline Age & 0.009 & 0.004 & 0.023 & & & & 0.011 & 0.004 & 0.012 & & & \\
\hline BMl & 0.004 & 0.006 & 0.525 & & & & 0.005 & 0.006 & 0.448 & & & \\
\hline CVD risk & -0.015 & 0.038 & 0.699 & & & & -0.014 & 0.039 & 0.731 & & & \\
\hline GDS & -0.007 & 0.015 & 0.654 & & & & -0.011 & 0.015 & 0.469 & & & \\
\hline APOE $\varepsilon 4$ & 0.303 & 0.056 & $<0.001$ & & & & 0.310 & 0.057 & $<0.001$ & & & \\
\hline rs151246 & 0.117 & 0.070 & 0.096 & & & & 0.064 & 0.175 & 0.716 & & & \\
\hline Latency & 0.009 & 0.002 & $<0.001$ & & & & 0.004 & 0.002 & 0.006 & & & \\
\hline INT & -0.009 & 0.003 & 0.002 & & & 0.039 & -0.008 & 0.007 & 0.294 & & & 0.004 \\
\hline \multicolumn{4}{|c|}{ Model summary: rs9951307 } & 0.186 & $<0.001$ & & & & & 0.166 & $<0.001$ & \\
\hline Age & 0.010 & 0.004 & 0.019 & & & & 0.010 & 0.004 & 0.013 & & & \\
\hline BMl & 0.003 & 0.006 & 0.607 & & & & 0.004 & 0.006 & 0.752 & & & \\
\hline CVD risk & -0.013 & 0.039 & 0.741 & & & & -0.012 & 0.039 & 0.752 & & & \\
\hline GDS & -0.007 & 0.015 & 0.658 & & & & -0.010 & 0.015 & 0.485 & & & \\
\hline APOE $\varepsilon 4$ & 0.312 & 0.056 & $<0.001$ & & & & 0.309 & 0.057 & $<0.001$ & & & \\
\hline rs9951307 & 0.015 & 0.070 & 0.831 & & & & 0.025 & 0.123 & 0.837 & & & \\
\hline Latency & 0.008 & 0.002 & 0.001 & & & & 0.004 & 0.002 & 0.006 & & & \\
\hline INT & -0.006 & 0.003 & 0.048 & & & 0.015 & -0.005 & 0.006 & 0.347 & & & 0.004 \\
\hline \multicolumn{4}{|c|}{ Model summary: rs 71353406} & 0.180 & $<0.001$ & & & & & 0.163 & $<0.001$ & \\
\hline Age & 0.010 & 0.004 & 0.023 & & & & 0.010 & 0.004 & 0.018 & & & \\
\hline BMl & 0.004 & 0.006 & 0.556 & & & & 0.005 & 0.006 & 0.401 & & & \\
\hline CVD risk & -0.008 & 0.039 & 0.833 & & & & -0.012 & 0.040 & 0.769 & & & \\
\hline GDS & -0.006 & 0.015 & 0.692 & & & & -0.009 & 0.015 & 0.552 & & & \\
\hline$A P O E \varepsilon 4$ & 0.298 & 0.058 & $<0.001$ & & & & 0.307 & 0.058 & $<0.001$ & & & \\
\hline rs71353406 & -0.063 & 0.069 & 0.362 & & & & 0.050 & 0.158 & 0.754 & & & \\
\hline Latency & 0.001 & 0.002 & 0.688 & & & & 0.004 & 0.002 & 0.022 & & & \\
\hline INT & 0.006 & 0.003 & 0.030 & & & 0.019 & 0.003 & 0.006 & 0.675 & & & 0.001 \\
\hline \multicolumn{4}{|c|}{ Model summary: rs3875089 } & 0.184 & $<0.001$ & & & & & 0.165 & $<0.001$ & \\
\hline Age & 0.010 & 0.004 & 0.019 & & & & 0.011 & 0.004 & 0.010 & & & \\
\hline BMl & 0.005 & 0.006 & 0.400 & & & & 0.004 & 0.006 & 0.458 & & & \\
\hline CVD risk & -0.016 & 0.039 & 0.683 & & & & -0.017 & 0.040 & 0.660 & & & \\
\hline GDS & -0.010 & 0.015 & 0.501 & & & & -0.012 & 0.015 & 0.426 & & & \\
\hline$A P O E \varepsilon 4$ & 0.310 & 0.057 & $<0.001$ & & & & 0.313 & 0.058 & $<0.001$ & & & \\
\hline rs3875089 & -0.050 & 0.074 & 0.497 & & & & -0.005 & 0.416 & 0.990 & & & \\
\hline Latency & 0.002 & 0.002 & 0.248 & & & & 0.004 & 0.002 & 0.008 & & & \\
\hline INT & 0.007 & 0.003 & 0.028 & & & 0.019 & 0.010 & 0.027 & 0.706 & & & 0.001 \\
\hline \multicolumn{4}{|c|}{ Model summary: rs491148 } & 0.185 & $<0.001$ & & & & & 0.193 & $<0.001$ & \\
\hline Age & 0.010 & 0.004 & 0.016 & & & & 0.012 & 0.004 & 0.005 & & & \\
\hline BMl & 0.005 & 0.006 & 0.360 & & & & 0.005 & 0.006 & 0.393 & & & \\
\hline
\end{tabular}


Table 3 continued

\begin{tabular}{|c|c|c|c|c|c|c|c|c|c|c|c|c|}
\hline & Dominant $^{\mathrm{a}}$ & & & & & & Recessi & & & & & \\
\hline & $\beta$ & s.e. & Sig. & $R^{2}$ & Sig. & $\Delta R^{2}$ & $\beta$ & s.e. & Sig. & $R^{2}$ & Sig. & $\Delta R^{2}$ \\
\hline CVD risk & -0.018 & 0.039 & 0.650 & & & & -0.017 & 0.039 & 0.657 & & & \\
\hline GDS & -0.011 & 0.015 & 0.450 & & & & -0.011 & 0.015 & 0.459 & & & \\
\hline APOE ع4 & 0.316 & 0.057 & $<0.001$ & & & & 0.320 & 0.057 & $<0.001$ & & & \\
\hline rs491148 & -0.035 & 0.075 & 0.639 & & & & -0.333 & 0.271 & 0.220 & & & \\
\hline Latency & 0.002 & 0.002 & 0.639 & & & & 0.004 & 0.001 & 0.014 & & & \\
\hline INT & 0.007 & 0.003 & 0.036 & & & 0.017 & 0.035 & 0.015 & 0.022 & & & 0.020 \\
\hline DURATION & & & & & & & & & & & & \\
\hline Model summary: & : rs72878776 & & & 0.149 & $<0.001$ & & & & & 0.126 & $<0.001$ & \\
\hline Age & 0.012 & 0.004 & 0.005 & & & & 0.011 & 0.004 & 0.010 & & & \\
\hline BMI & 0.005 & 0.006 & 0.370 & & & & 0.004 & 0.006 & 0.520 & & & \\
\hline CVD risk & -0.023 & 0.040 & 0.565 & & & & -0.009 & 0.040 & 0.816 & & & \\
\hline GDS & -0.007 & 0.015 & 0.662 & & & & -0.003 & 0.015 & 0.838 & & & \\
\hline APOE $\varepsilon 4$ & 0.289 & 0.058 & $<0.001$ & & & & 0.283 & 0.059 & $<0.001$ & & & \\
\hline rs12968026 & 0.807 & 0.352 & 0.023 & & & & 0.065 & 0.715 & 0.928 & & & \\
\hline Duration & 0.026 & 0.023 & 0.251 & & & & 0.005 & 0.021 & 0.817 & & & \\
\hline INT & -0.104 & 0.049 & 0.034 & & & 0.019 & -0.010 & 0.105 & 0.923 & & & $<0.001$ \\
\hline Model summary: & : rs2339214 & & & 0.132 & $<0.001$ & & & & & 0.174 & $<0.001$ & \\
\hline Age & 0.010 & 0.004 & 0.018 & & & & 0.011 & 0.004 & 0.009 & & & \\
\hline BMl & 0.005 & 0.006 & 0.403 & & & & 0.004 & 0.006 & 0.507 & & & \\
\hline CVD risk & -0.011 & 0.041 & 0.796 & & & & -0.009 & 0.040 & 0.819 & & & \\
\hline GDS & -0.005 & 0.016 & 0.774 & & & & -0.008 & 0.015 & 0.595 & & & \\
\hline APOE \&4 & 0.302 & 0.059 & $<0.001$ & & & & 0.307 & 0.058 & $<0.001$ & & & \\
\hline rs2339214 & 0.056 & 0.324 & 0.864 & & & & -0.993 & 0.329 & 0.003 & & & \\
\hline Duration & 0.014 & 0.038 & 0.714 & & & & -0.031 & 0.024 & 0.197 & & & \\
\hline INT & -0.009 & 0.045 & 0.850 & & & $<0.001$ & 0.149 & 0.047 & 0.002 & & & 0.041 \\
\hline Model Summary & : rs491148 & & & & 0.156 & $<0.001$ & & & & & 0.146 & \\
\hline Age & 0.011 & 0.004 & 0.007 & & & & 0.012 & 0.004 & 0.005 & & & \\
\hline BMl & 0.005 & 0.006 & 0.377 & & & & 0.004 & 0.006 & 0.736 & & & \\
\hline CVD risk & -0.023 & 0.040 & 0.565 & & & & -0.016 & 0.040 & 0.684 & & & \\
\hline GDS & -0.012 & 0.015 & 0.419 & & & & -0.008 & 0.015 & 0.574 & & & \\
\hline APOE $\varepsilon 4$ & 0.317 & 0.058 & $<0.001$ & & & & 0.316 & 0.059 & $<0.001$ & & & \\
\hline rs491148 & 0.707 & 0.320 & 0.028 & & & & -0.135 & 0.662 & 0.839 & & & \\
\hline Duration & 0.030 & 0.024 & 0.202 & & & & 0.005 & 0.021 & 0.819 & & & \\
\hline INT & -0.090 & 0.045 & 0.045 & & & 0.016 & 0.053 & 0.097 & 0.584 & & & 0.001 \\
\hline
\end{tabular}

${ }^{a}$ Genetic models: Dominant (heterozygote/homozygote for the minor allele (Mm or MM) vs. homozygote for the major allele (mm)), Recessive (homozygote for the minor allele (MM) vs. heterozygote/homozygote for the major allele (Mm/mm)); $\beta$ coefficient of predictors; Sig $p$-value; $R^{2}$ coefficient of multiple determination; $\Delta R^{2}$ multiple correlation coefficient $(R)$ squared change; $A P O E$ Apolipoprotein $\mathrm{E} \varepsilon 4$ allele carriage (presence/absence); $B M I$ body mass index; $C V D$ risk cardiovascular disease risk; GDS Geriatric Depression Scale; INT Interaction (Sleep Latency/Duration $\times$ model summary SNP). Models where the interaction term (INT) resulted in a statistically significant $R^{2}$-change $(p<0.05)$ are indicated (bolded)

Model summary statistics for significant Aquaporin-4 (AQP4) reference single-nucleotide polymorphism (SNP) markers (rs) 


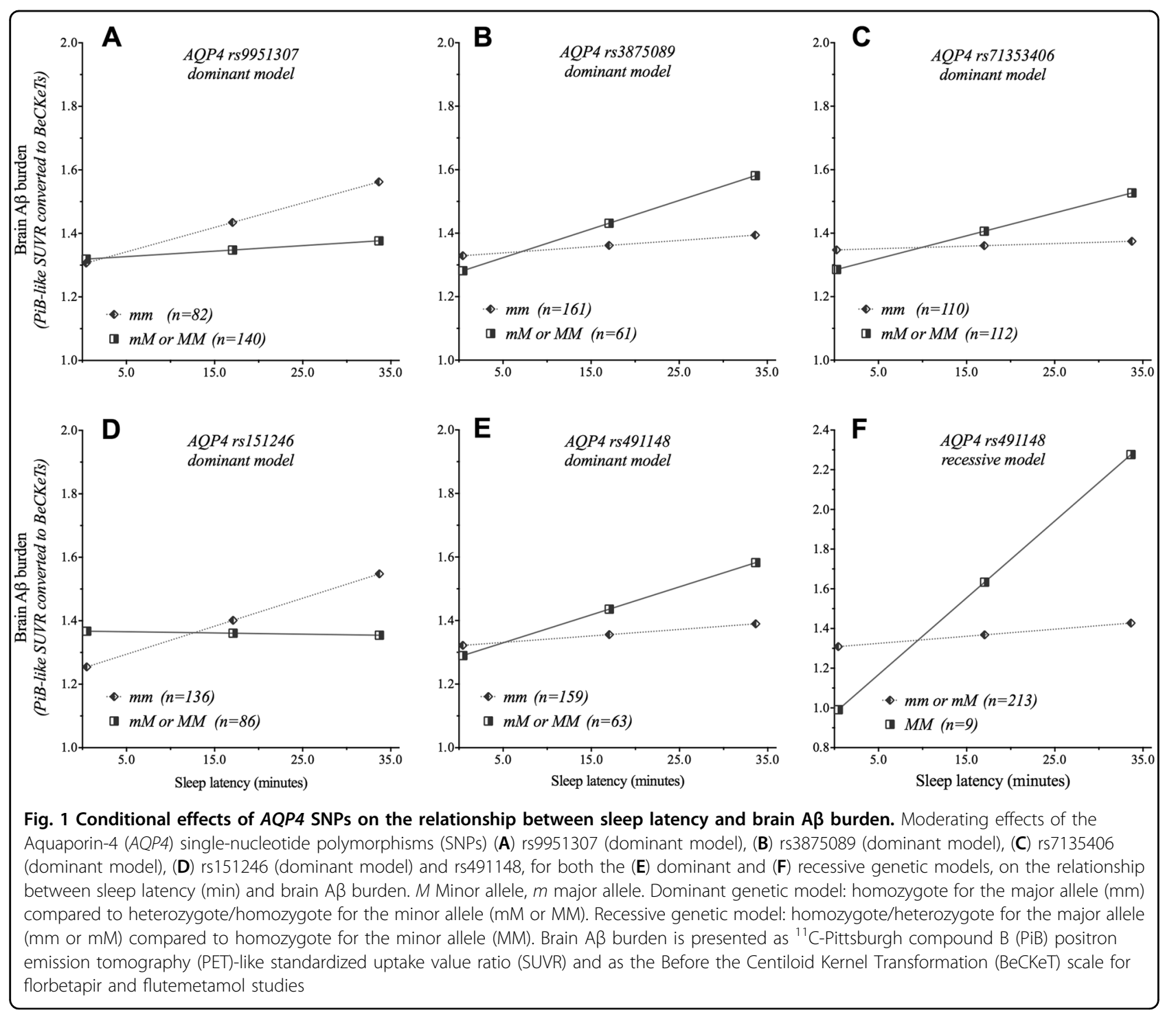

$=184$ ) of AIBL participants included in the current study $(n=222)^{12}$. In this prior study, no moderation of the sleep latency-A $\beta$ relationship by $A P O E$ genotype was observed. However, we report in the current study that moderation of this relationship occurs due to variants in a gene encoding a key component of the postulated glymphatic system: Specifically, AQP4, which encodes for AQP4, an astrocytic end-feet expressed water-channel protein postulated to be involved in glymphatic-mediated clearance of $\mathrm{A} \beta^{18}$.

Our data suggests that genetic variation in $A Q P 4$, specifically rs72878776, is associated with altered, selfreported, 'overall' sleep quality (PSQI Total score), with individuals homozygous for the AQP4 rs72878776-A allele reporting worse overall sleep compared to those with a different genotype. This SNP is in the 5-prime untranslated region ( $\left.5^{\prime} \mathrm{UTR}\right)$ of the $A Q P 4$ gene and may be of functional relevance through potentially influencing gene transcription, via modification (creation or deletion) of transcription factor binding sites. This is supported by evidence compiled in the RegulomeDB ${ }^{33}$. Specifically, the potential binding of transcriptional regulators such as REST, TRIM28, CTBP2, and ZNF263 are predicted to be affected by this variant. Analysis of the LD structure of the AQP4 gene for linkage of rs72878776, with other variants with potential functional implications, revealed it to tag rs35248760, a synonymous SNP in exon 1. While rs35248760 does not appear itself to be a SNP that impacts functionality of the protein it encodes, it cannot be ruled out that rs72878776 may also be in linkage with rare non-synonymous variants in exon 1 .

Five AQP4 SNPs (rs9951307, rs7135406, rs3875089, rs151246, and rs491148) in the dominant models, had significant interactions with self-reported sleep latency 


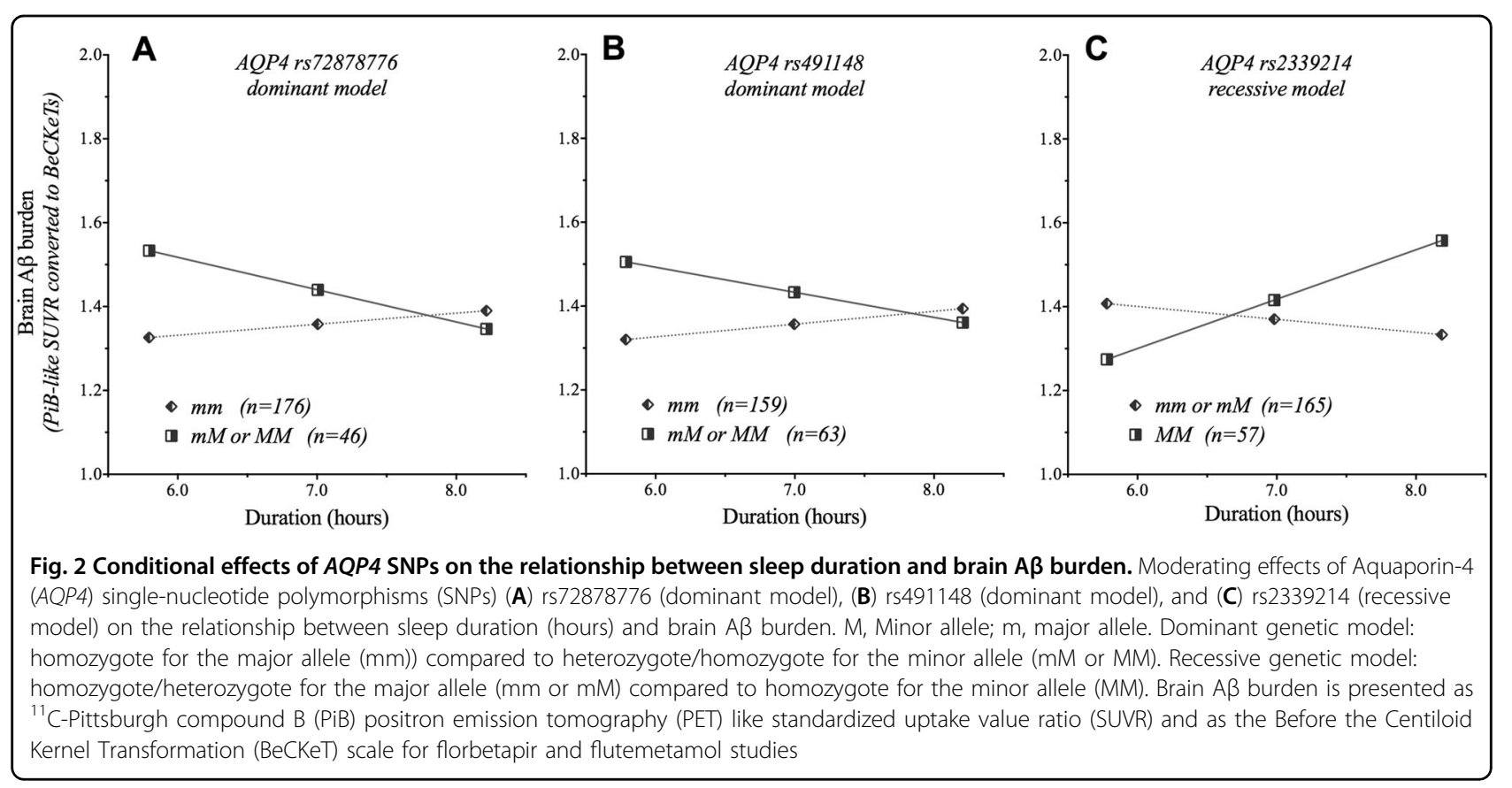

and the resultant effect on brain $\mathrm{A} \beta$ burden. The impact of rs491148 was observably stronger in homozygotes, suggesting a potential gene-dosage effect for the minor allele (rs491148-G). Specifically, carriage of at least one copy of the rs491148-G allele was associated with a PiBlike SUVR approaching 1.6 when time to fall asleep reached $35 \mathrm{~min}$, while homozygosity of the G-allele, albeit in only 9 individuals, was associated with $A \beta$ burdens approaching 2.3 SUVR/BeCKeT at $35 \mathrm{~min}$ latency-a level of brain $A \beta$ usually associated with a clinical diagnosis of mild $\mathrm{AD}^{7}$. Of note, three of these $A Q P 4$ variants; rs9951307 (D' 0.99, $r^{2} 0.07$ ), rs3875089 (D' 1.00, $r^{2} 0.64$ ), and rs491148 ( $\left.\mathrm{D}^{\prime} 0.94, r^{2} 0.49\right)$, are in strong $\mathrm{LD}$, but have reduced correlation, with the aforementioned $5^{\prime} \mathrm{UTR}$ rs72878776. These $A Q P 4$ SNP-sleep latency findings support previous studies which have reported an association of sleep latency with brain $A \beta^{10,12}$. The current study adds evidence that this relationship is likely moderated by genetic variation in the gene encoding the Aquaporin-4 water-channel protein, which is proposed to be involved in glymphatic-mediated $A \beta$ clearance during sleep $^{34}$. Accordingly, those $A Q P 4$ SNPs that impact the relationship between sleep latency and $A \beta$ may predispose those individuals to suboptimal sleep parameters due to higher $\mathrm{A} \beta$ burden. Alternatively, as a bi-directional relationship between sleep and $A \beta$ has also been postulated ${ }^{3,5}$, it is conceivable that suboptimal sleep contributes to higher brain $\mathrm{A} \beta$ burden, particularly in those potentially genetically predisposed to poorer functioning of $A \beta$ clearance mechanisms. However, it is also conceivable that the association between $A Q P 4$ variants and sleep quality observed in this study may potentially be attributed to mechanisms unrelated to $A \beta$ dynamics. For example, any impact of genetic variation on expression of AQP4 may, through disrupted water molecule conduct, affect intracellular ionic homeostasis, resulting in impaired cellular function or even death. Since AQP4 is enriched in the glial cells of periventricular regions in the hypothalamus, where hypocretin (orexin)-containing neurons are primarily located, it is conceivable that impaired glial function in these regions may result in secondary neuronal damage leading to impaired sleep regulation through subtle hypocretin deficiency. This is observed to a larger extent in cases of narcolepsy where the presence of anti-AQP4 antibodies is observed ${ }^{35}$.

We also identified three AQP4 SNPs that interacted with sleep duration to have a moderating effect on levels of $A \beta$ in the brain. Two of these, rs72878776 and rs491148, were also associated with overall sleep quality, and moderation of the relationship between sleep latency and $\mathrm{A} \beta$ burden in this study, respectively. With respect to sleep duration, carriage of the minor allele for both rs72878776 and rs491148, was associated with higher $A \beta$ burden with a shorter duration of sleep. However, the opposite was observed for the final variant, rs2339214, Specifically, longer sleep duration (rather than shorter duration) was associated with higher brain $\mathrm{A} \beta$ (PiB-like SUVR $\sim 1.6,>8 \mathrm{~h}$ sleep duration) in individuals homozygote for the minor allele, rs2339214-A. To our knowledge there is no previous report in the literature of a bimodal relationship between sleep duration and brain $\mathrm{A} \beta$ burden. However, there is evidence in the literature that such a bimodal relationship exists between sleep duration and cognition. Specifically, both short and long sleep 
duration are purported to contribute to poorer cognitive function and increased risk of cognitive impairment and $\mathrm{AD}$ compared to intermediate sleep duration ${ }^{36-38}$.

In a recent study by Burfeind and colleagues, two of the AQP4 SNPs described in the current study, rs9951307 and rs3875089, were reported to be associated with altered trajectories of cognitive decline. We have previously reported in the AIBL study that $A \beta$ is associated with cognitive decline ${ }^{39-43}$ and, as discussed above, suboptimal sleep has also been associated with poorer cognitive function $^{36-38}$. As such, it is plausible that the association of $A Q P 4$ genetic variation with cognitive decline described by Burfeind et al., may be mediated through the impact of $A Q P 4$ on brain $A \beta$. Interestingly, the association with cognitive decline reported by the authors was limited to those with an established clinical diagnosis of $\mathrm{AD}$, in whom a high $A \beta$ burden would be expected, and was not observed in the 'Pre-AD' group. Further, while post mortem evaluation of $\mathrm{AD}$ pathology was undertaken, global brain $\mathrm{A} \beta$ burden was not evaluated pre-mortem; additional investigation is therefore required to fully elucidate the AQP4-cognition relationship, particularly during the preclinical stages of $\mathrm{AD}$ and with respect to global brain $\mathrm{A} \beta$ burden.

The functional implication of the genetic variants reported herein remain poorly understood and it is clear that further study is required to understand the mechanism that underpins these associations. While there is in silico evidence to suggest that some variants may impact the binding of transcription factors, there are other putative mechanisms that may play a role in the relationship between $A Q P 4$ genetic variation and $\mathrm{A} \beta$ clearance. For example, several associated SNPs are physically, or in LD with other SNPs, located in the putative promoter region of the AQP4-M23 isoform (M23), the smaller of two isoforms of AQP4 ${ }^{44}$, the other being AQP4-M1 (M1). It has been reported that an increased ratio of M23:M1 isoforms occurs in $\mathrm{AD}$ and is associated with altered perivascular localization of $\mathrm{AQP} 4^{21}$. With this loss of perivascular localization, a concomitant worsening of $A \beta$ plaque burden was observed ${ }^{21}$ suggesting that genetic variation that alters isoform relative expression may in turn impact $\mathrm{A} \beta$ clearance. Further to this, microRNA mediated regulation of AQP4 expression, particularly of the M1 isoform, has been reported ${ }^{45}$. However, of the SNPs reported to moderate sleep-A $\beta$ relationships in this study, none were located within the putative microRNA binding sites identified previously ${ }^{45}$, or within the putative M1 promoter region itself. More recently, De Bellis et al. ${ }^{46}$ have demonstrated that in addition to M1 and M23 isoforms, AQP4 may be subject to translational readthrough to generate functionally significant C-terminal extended isoforms, termed AQP4ex. However, of all the associated variants reported in this study, only rs9951307 is located at the C-terminal end of $A Q P 4$, and is $\sim 15 \mathrm{~kb}$ downstream of the AQP4 UGA canonical stop-codon. Further, this variant does not tag any genetic variants in the vicinity of the stop-codon, although linkage with one or multiple rare-variants in this region cannot be ruled out. Additional study is required to determine the impact of any of these variants on AQP4M1, AQP4-M23, or AQP4ex isoform expression.

While the findings of this study are novel and suggest that genetic variation of $A Q P 4$ moderates the relationship between sleep parameters and brain $A \beta$ burden, there are some limitations that need to be considered. First, this study was observational and utilized a cross-sectional retrospective design; consequently, no conclusions regarding temporal or causal relationships can be drawn. Second, a subjective sleep measure was utilized which relies on the accuracy and fidelity of the respondents. Utilization of an objective measure of sleep such as actigraphy or polysomnography would circumvent the limitation of self-report. Moreover, use of polysomnography, the 'gold standard' in differentiating sleep from wake, and in identifying sleep stages, would provide detail regarding the association of sleep architecture with brain A $\beta$. However, the PSQI has demonstrated internal consistent reliability and construct validity ${ }^{47}$ and is justified in this study due to its cost effectiveness and ease of administration to a large cohort. Third, the brain imaging and PSQI administration were completed on separate days; however, $A \beta$ deposition is a relatively slow process, occurring over many years ${ }^{7}$, and sleep habits are usually chronic, particularly in the age group studied. Nevertheless, it is acknowledged that administration of the PSQI at multiple time points would provide a longer window of assessment of sleep parameters and therefore may be more informative. Finally, any inferences of potential glymphatic clearance underpinning the association of $A Q P 4$ genetic variation with $A \beta$ clearance from the brain in humans and the potential functional implications of genetic variation on isoform-specific expression are speculative and require further functional studies to elucidate.

Our study adds weight to the proposition that paravascular clearance, encompassing the postulated glymphatic system, is a potential biological mechanism that underpins $\mathrm{A} \beta$ clearance from the brain ${ }^{14}$. Whether other genetic factors beyond $A P O E$ and $A Q P 4$, examined here, may likewise moderate the relationship between sleep parameters and $\mathrm{AD}$ characteristics remains to be determined, however, the current study provides evidence to support future investigation of such interactions. Prospectively, the results of this study provide a greater understanding of what factors may impact on the sleep$\mathrm{AD}$ phenotype relationship, and support the notion that establishing interventions targeted at improving sleep parameters maybe beneficial for positively modulating 
cerebral $\mathrm{A} \beta$ levels and thus, potentially delaying $\mathrm{AD}$ onset. Indeed, findings from this study could be used to both stratify retrospective analysis of existing datasets, or perhaps more importantly, to derive tailored AD intervention strategies based on the genetics of the individual: e.g. a sleep-specific intervention targeted at reducing sleep latency may be most beneficial to individuals who are genetically predisposed to a heightened impact of latency on pathological or clinical outcomes. Overall, the data from this study provide evidence that genetic variation in the cerebrally expressed water-channel protein, Aquaporin-4, moderates the relationship between sleep and brain $\mathrm{A} \beta$ burden.

\section{Acknowledgements}

Funding for the AIBL study was provided in part by the study partners (Commonwealth Scientific Industrial and Research Organization (CSIRO), Edith Cowan University (ECU), Mental Health Research institute (MHRI), National Ageing Research Institute (NARI), Austin Health, CogState Ltd.). The AIBL study has also received support from the National Health and Medical Research Council (NHMRC) and the Dementia Collaborative Research Centres program (DCRC2), as well as funding from the Science and Industry Endowment Fund (SIEF) and the Co-operative Research Centre (CRC) for Mental Health — funded through the CRC Program (Grant ID:20100104), an Australian Government Initiative. We thank all those who took part as subjects in the study for their commitment and dedication to helping advance research into the early detection and causation of AD.

\section{Conflict of interest}

C.L.M. is an advisor to Prana Biotechnology Ltd and a consultant to Eli Lilly. P.M. is a full-time employee of Cogstate Ltd. D.A. has served on scientific advisory boards for Novartis, Eli Lilly, Janssen, and Pfizer Inc. R.N.M. is a consultant to Alzhyme. C.C.R. has served on scientific advisory boards for Bayer Pharma, Elan Corporation, GE Healthcare and AstraZeneca; has received speaker honoraria from Bayer Pharma and GE Healthcare; and has received research support from Bayer Pharma, GE Healthcare, Piramal Lifesciences and Avid

Radiopharmaceuticals. H.R.S. has received/is receiving remunerations from the Australian Alzheimer's Research Foundation for working on several of their clinical trials associated with Takeda, Merck and AstraZeneca and Eli Lilly. VLV served as a consultant for Bayer Pharma; and received research support from a NEDO grant from Japan. S.M.L. has previously been a paid consultant to Alzhyme. The remaining authors declare that they have no conflict of interest.

\section{Publisher's note}

Springer Nature remains neutral with regard to jurisdictional claims in published maps and institutional affiliations.

Supplementary Information accompanies this paper at (https://doi.org/ 10.1038/s41398-018-0094-x).

Received: 9 September 2017 Revised: 22 November 2017 Accepted: 30 December 2017

Published online: 26 February 2018

\section{References}

1. Peter-Derex, L., Yammine, P., Bastuji, H. \& Croisile, B. Sleep and Alzheimer's disease. Sleep. Med. Rev. 19, 29-38 (2015).

2. Wolkove, N., Elkholy, O., Baltzan, M. \& Palayew, M. Sleep and aging: 1. Sleep disorders commonly found in older people. Can. Med. Assoc. J. 176 1299-1304 (2007).

3. Ju, Y. E. S., Lucey, B. P. \& Holtzman, D. M. Sleep and Alzheimer disease pathology—A bidirectional relationship. Nat. Rev. Neurol. 10, 115-119 (2014).
4. Holth, J. K. Patel, T. K. \& Holtzman, D. M. Sleep in Alzheimer's disease-beyond amyloid. Neurobiol. Sleep. Circ. Rhythms 2, 4-14 (2017).

5. Brown, B. M., Rainey-Smith, S. R., Bucks, R. S., Weinborn, M. \& Martins, R. N. Exploring the bi-directional relationship between sleep and beta-amyloid. Curr. Opin. Psychiatry 29, 397-401 (2016).

6. Selkoe, D. J. \& Hardy, J. A. The amyloid hypothesis of Alzheimer's disease at 25 years. EMBO Mol. Med. 8, 595-608 (2016).

7. Villemagne, V. L. et al. Amyloid $\beta$ deposition, neurodegeneration, and cognitive decline in sporadic Alzheimer's disease: A prospective cohort study. Lancet Neurol. 12, 357-367 (2013).

8. ENTP, Bakker et al. Lymphatic clearance of the brain: Perivascular, paravascular and significance for neurodegenerative diseases. Cell Mol. Neurobiol. 36, 181-194 (2016).

9. Xie, L. et al. Sleep drives metabolite clearance from the adult brain. Science 342, 373-377 (2013).

10. Branger, P. et al. Relationships between sleep quality and brain volume, metabolism, and amyloid deposition in late adulthood. Neurobiol. Aging 41, 107-114 (2016).

11. Kang, J. E. et al. Amyloid-beta dynamics are regulated by orexin and the sleepwake cycle. Science 326, 1005-1007 (2009).

12. Brown, B. M. et al. The relationship between sleep quality and brain amyloid burden. Sleep 39, 1063-1068 (2016).

13. Kress, B. T. et al. Impairment of paravascular clearance pathways in the aging brain. Ann. Neurol. 76, 845-861 (2014).

14. Iliff, J. J. et al. A paravascular pathway facilitates CSF flow through the brain parenchyma and the clearance of interstitial solutes, including amyloid $\beta$. Sci. Transl. Med. 4, 147ra111-147ra111 (2012).

15. Jessen, N. A., Munk, A. S. F., Lundgaard, I. \& Nedergaard, M. The glymphatic system: A beginner's guide. Neurochem. Res. 40, 2583-2599 (2015).

16. Lundgaard, I. et al. Glymphatic clearance controls state-dependent changes in brain lactate concentration. J. Cereb. Blood Flow. Metab. 37, 2112-2124 (2016).

17. Yang, W. et al. Aquaporin-4 mediates astrocyte response to $\beta$-amyloid. Mol. Cell Neurosci. 49, 406-414 (2012).

18. Xiao, M. \& Hu, G. Involvement of aquaporin 4 in astrocyte function and neuropsychiatric disorders. CNS Neurosci. Ther. 20, 385-390 (2014).

19. Moftakhar, P., Lynch, M. D., Pomakian, J. L. \& Vinters, H. V. Aquaporin expression in the brains of patients with or without cerebral amyloid angiopathy. J. Neuropathol. Exp. Neurol. 69, 1201-1209 (2010).

20. Hoshi, A. et al. Characteristics of aquaporin expression surrounding senile plaques and cerebral amyloid angiopathy in Alzheimer disease. J. Neuropathol. Exp. Neurol. 71, 750-759 (2012).

21. Zeppenfeld, D. M. et al. Association of perivascular localization of aquaporin-4 with cognition and Alzheimer disease in aging brains. JAMA Neurol. 74, 91-99 (2017).

22. Sorani, M. D. et al. Novel variants in human Aquaporin-4 reduce cellular water permeability. Hum. Mol. Genet. 17, 2379-2389 (2008).

23. Ellis, K. A. et al. The Australian Imaging, Biomarkers and Lifestyle (AIBL) study of aging: Methodology and baseline characteristics of 1112 individuals recruited for a longitudinal study of Alzheimer's disease. Int. Psychogeriatr. 21, 672-687 (2009).

24. Buysse, D. J., Reynolds, C. F., Monk, T. H., Berman, S. R. \& Kupfer, D. J. The Pittsburgh sleep quality index: A new instrument for psychiatric practice and research. Psychiatry Res. 28, 193-213 (1989).

25. Clark, C. M. et al. Use of Florbetapir-PET for imaging $\beta$-amyloid pathology. JAMA 305, 275-283 (2011).

26. Rowe, C. C. et al. Amyloid imaging results from the Australian Imaging, Biomarkers and Lifestyle (AIBL) study of aging. Neurobiol. Aging 31, 1275-1283 (2010).

27. Vandenberghe, R. et al. 18F-Flutemetamol amyloid imaging in Alzheimer disease and mild cognitive impairment: A phase 2 trial. Ann. Neurol. 68, 319-329 (2010).

28. Bourgeat, P. et al. Comparison of MR-less PiB SUVR quantification methods. Neurobiol. Aging 36, S159-S166 (2015).

29. Villemagne, V. L. et al. En attendant centiloid. Adv. Res. 2, 723-729 (2014)

30. Benjamini, Y. \& Yekutieli, D. The control of the false discovery rate in multiple testing under dependency. Ann. Stat. 29, 1165-1188 (2001).

31. Hayes, A. F. Introduction to Mediation, Moderation, and Conditional Process Analysis: A Regression-based Approach.. (The Guilford Press, New York, NY, 2013). 
32. Hayes, A. F. \& Rockwood N. J. Regression-based statistical mediation and moderation analysis in clinical research: observations, recommendations, and implementation. Behav. Res. Ther. 98, 39-57 (2017).

33. Boyle, A. P. et al. Annotation of functional variation in personal genomes using RegulomeDB. Genome Res. 22, 1790-1797 (2012).

34. Mander, B. A., Winer, J. R., Jagust, W. J. \& Walker, M. P. Sleep: A novel mechanistic pathway, biomarker, and treatment target in the pathology of Alzheimer's disease? Trends Neurosci. 39, 552-566 (2016).

35. Kanbayashi, T. et al. Symptomatic narcolepsy in patients with neuromyelitis optica and multiple sclerosis: new neurochemical and immunological implications. Arch. Neurol. 66, 1563-1566 (2009).

36. $\mathrm{Xu}, \mathrm{L}$. et al. Short or long sleep duration is associated with memory impairment in older Chinese: The Guangzhou Biobank Cohort Study. Sleep 34, 575-580 (2011).

37. Potvin, $\mathrm{O}$. et al. Sleep quality and 1-year incident cognitive impairment in community-dwelling older adults. Sleep 35, 491-499 (2012).

38. Schmutte, T. et al. The relation between cognitive functioning and selfreported sleep complaints in nondemented older adults: Results from the Bronx aging study. Behav. Sleep Med. 5, 39-56 (2007).

39. Villemagne, $\mathrm{V}$. L. et al. Longitudinal assessment of Abeta and cognition in aging and Alzheimer disease. Ann. Neurol. 69, 181-192 (2011).
40. Villemagne, V. L. et al. Amyloid beta deposition, neurodegeneration, and cognitive decline in sporadic Alzheimer's disease: a prospective cohort study. Lancet Neurol. 12, 357-367 (2013).

41. Lim, Y. Y. et al. BDNF Val66Met, Abeta amyloid, and cognitive decline in preclinical Alzheimer's disease. Neurobiol. Aging 34, 2457-2464 (2013).

42. Lim, Y. Y. et al. APOE and BDNF polymorphisms moderate amyloid betarelated cognitive decline in preclinical Alzheimer's disease. Mol. Psychiatry 20, 1322-1328 (2015).

43. Lim, Y. Y. et al. APOE epsilon4 moderates amyloid-related memory decline in preclinical Alzheimer's disease. Neurobiol. Aging 36, 1239-1244 (2015).

44. Nagelhus, E. A. \& Ottersen, O. P. Physiological roles of aquaporin-4 in brain. Physiol. Rev. 93, 1543-1562 (2013).

45. Sepramaniam, S., Ying, L. K., Armugam, A., Wintour, E. M. \& Jeyaseelan, K MicroRNA-130a represses transcriptional activity of aquaporin $4 \mathrm{M} 1$ promoter. J. Biol. Chem. 287, 12006-12015 (2012).

46. De Bellis, M. et al. Translational readthrough generates new astrocyte AQP4 isoforms that modulate supramolecular clustering, glial endfeet localization and water transport. Glia 65, 790-803 (2017).

47. Mollayeva, T. et al. The Pittsburgh Sleep Quality index as a screening tool for sleep dysfunction in clinical and non-clinical samples: A systematic review and meta-analysis. Sleep Med. Rev. 25, 52-73 (2016). 